\title{
Pseudoprogresión cutánea por inmunoterapia en melanoma metastásico
}

\author{
Cutaneous pseudoprogression due to \\ immunotherapy in metastatic melanoma
}

\author{
Ivonne Salcedo Sullk, ${ }^{*}$ Alejandro Noguez Ramos, ${ }^{*}$, Lorena López Zepeda, ${ }^{*}$ José Fabián Martínez-Herrera ${ }^{*}, \S$
}

Citar como: Salcedo SI, Noguez RA, López ZL, Martínez-Herrera JF. Pseudoprogresión cutánea por inmunoterapia en melanoma metastásico. An Med ABC. 2021; 66 (4): 288-291. https://dx.doi.org/10.35366/102929

\section{RESUMEN}

El melanoma representa menos de 5\% de los tumores de la piel. En etapas avanzadas el pronóstico es malo, la supervivencia global alcanza de seis a nueve meses. Con la introducción de los bloqueadores de los puntos de control inmunológicos el panorama de estos pacientes ha cambiado, con incremento en los desenlaces oncológicos y mejor tolerancia a los tratamientos. La pseudoprogresión es un tipo de respuesta poco frecuente en estos pacientes y representa un desafío para el médico oncólogo. Presentación del caso: Mujer de 80 años con melanoma nodular en pierna izquierda, Breslow $4.5 \mathrm{~mm}$, Clark IV, etapa clínica IIB, recurrente metastásico ganglionar pretibial izquierda, con periodo libre de enfermedad de 15 meses. El tratamiento a la recurrencia fue con control local quirúrgico; sin embargo, los estudios de imagen postquirúrgicos mostraron actividad tumoral local y pulmonar, por lo que inició inmunoterapia a base de pembrolizumab. $\mathrm{Al}$ cuarto ciclo mostró aumento del diámetro longitudinal y elevación de las lesiones que fueron catalogadas como pseudoprogresión, razón por la que continuó el tratamiento. Cuatro aplicaciones después, presentó disminución de los diámetros de las lesiones. Posteriormente se realizó control de la lesión pulmonar con radiocirugía. A la fecha, la paciente ha recibido 22 ciclos manteniendo la respuesta parcial obtenida y excelente calidad de vida. La pseudoprogresión es un tipo de respuesta que debemos aprender a evaluar en pacientes con melanoma en tratamiento con inmunoterapia, antes de considerar un cambio de fármacos.

Palabras clave: Melanoma, inmunoterapia, pseudoprogresión.

\section{ABSTRACT}

The melanoma represents less than 5\% of skin tumors. In advanced stages the prognosis is poor, overall survival reaches 6-9 months. Because of the introduction of immune checkpoint blockers the landscape of these patients has changed, pseudoprogression is a rare type of response in these patients and represents a challenge for the medical oncologist. Case report: An 80-year-old woman with nodular melanoma on the left leg, Breslow $4.5 \mathrm{~mm}$, Clark IV, clinical stage IIB, recurrent metastatic in left pretibial lymph node, with a disease-free period of 15 months. Treatment at recurrence was surgical control; nevertheless, post-surgical imaging studies showed local and pulmonary tumor activity, therefore pembrolizumab-based immunotherapy was started. At fourth cycle, she presented an increase in the longitudinal diameter and elevation of the lesions that were classified as pseudoprogression, so she continued the treatment. Four applications later, the diameter of the lesions decreased. Subsequently, the lung lesion was controlled with radiosurgery. At this time, the patient has received 22 cycles and continues with stable disease. Pseudoprogression is a type of response that we must learn to evaluate in patients with melanoma undergoing immunotherapy, before considering a change in drugs.

Keywords: Melanoma, immunotherapy, pseudoprogression.
* Departamento de Oncología Médica. Centro Médico ABC, Ciudad de México.

₹ Departamento de tumores gastrointestinales, Instituto Nacional de Ciencias Médicas y Nutrición «Salvador Zubirán» (INCMNSZ), Ciudad de México.

$\S$ Latin American Network for Cancer Research (LAN-CANCER), Lima, Perú.

\section{Correspondencia:}

Ivonne Salcedo Sullk

E-mail: salcedo_ivonne@ hotmail.com

Recibido: 26/05/2021. Aceptado: 08/08/2021. 


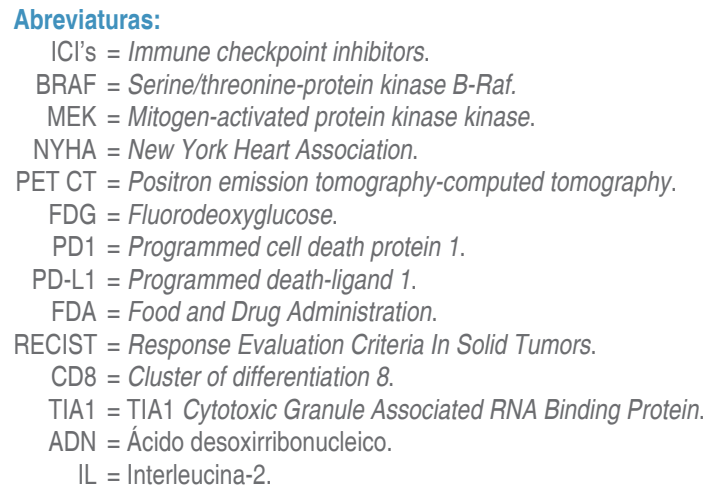

\section{INTRODUCCIÓN}

El melanoma es un tumor que surge de los melanocitos; cada año se diagnostican a nivel mundial más de 200,000 casos y cerca de 55,000 muertes anuales, siendo la principal causa de muerte por cáncer de piel. ${ }^{1,2}$ En Estados Unidos se diagnosticaron 91,000 casos en 2018 que representan 5\% de todos los diagnósticos de cáncer. ${ }^{3}$ En México, los datos disponibles son escasos debido a una deficiente recolección de información sobre el cáncer, pero se sabe que ocupa el séptimo lugar de todos los diagnósticos de cáncer. ${ }^{4}$

Con relación a la mortalidad, según el estudio publicado por Hernández, la tasa de mortalidad aumentó a $78 \%$ en un periodo de 1998 a 2016, independientemente del sexo o la edad del paciente al momento del diagnóstico; esto puede estar relacionado con el tiempo hasta el diagnóstico, además del acceso limitado a los nuevos tratamientos disponibles para tratar esta neoplasia. ${ }^{3,4} \mathrm{El}$ pronóstico de estos pacientes depende del estadio clínico en el que sean diagnosticados. En los países desarrollados por lo general se diagnóstica en etapas tempranas, a diferencia de México, donde la mayoría se detecta en estadios localmente avanzados hasta en $80 \% .{ }^{4}$ A nivel mundial, la supervivencia ha mejorado en los últimos años con la introducción de nuevos fármacos como los inhibidores de puntos de control inmunológicos (ICI's) o la terapia dirigida anti-BRAF/MEK, cuando anteriormente con el uso de quimioterapia sólo se alcanzaba alrededor de $47 \%$ al año, con una supervivencia global de nueve meses. ${ }^{5,6}$ Hoy en día, con los nuevos tratamientos con pembrolizumab se alcanza una supervivencia global de 33 meses y de $39 \%{ }^{7}$ a cinco años, mientras que con nivolumab se logra una supervivencia de $72 \%$ a nueve años. ${ }^{8}$

La pseudoprogresión se define como el aumento inicial del tamaño del tumor primario o la aparición de nuevas lesiones, seguido de la regresión de todas, siempre relacionada con la administración de inhibidores de puntos de control. Cabe mencionar que es un fenómeno raro que tiene una incidencia aproximada de $10 \%$, pero su identificación es de gran relevancia, ya que puede determinar el curso terapéutico de los pacientes, por lo que existen diferentes propuestas para su correcto diagnóstico que hasta ahora no han sido estandarizadas. Aquí presentamos un caso de melanoma avanzado irresecable con pseudoprogresión.

\section{CASO CLÍNICO}

Presentamos a una mujer de 80 años con antecedentes de cáncer epidermoide resecado en 2012 sin evidencia de recurrencia a la fecha; además de diabetes tipo 2, insuficiencia cardiaca NYHA II, portadora de marcapaso permanente por arritmia no especificada y neumopatía intersticial.

Inicialmente fue diagnosticada con melanoma invasor del tipo nodular en la pierna izquierda, Breslow $4.5 \mathrm{~mm}$, Clark IV, sin ulceración, márgenes libres, índice mitótico 12 por $\mathrm{mm}^{2}$, sin microsatelitosis, sin invasión vascular o perineural, con ganglio centinela negativo, etapa clínica IIB. Fue sometida a resección y después de cirugía se mantuvo en vigilancia activa con revisión clínica e imagen (PET CT) sin evidencia de actividad tumoral.

Después de 15 meses de periodo libre de enfermedad se documentó recurrencia ganglionar pretibial izquierda, por lo que fue sometida a escisión amplia en la región pretibial izquierda con linfadenectomía inguinal superficial izquierda y pélvica profunda con reconstrucción e injerto autólogo. El estudio de patología reportó melanoma recurrente que afectaba hasta el tejido celular subcutáneo, sin ulceración de la epidermis, bordes negativos, invasión linfática presente, sin invasión perineural, con 40 ganglios positivos de 42 resecados, con extensión extraganglionar multifocal.

Se realizó PET CT con FDG que mostró lesiones nodulares en tejido celular subcutáneo, ganglionares femorales profundos y cadena iliaca, además de nódulo pulmonar con actividad metabólica, por lo que inició inmunoterapia con pembrolizumab $2 \mathrm{mg} / \mathrm{kg}$ cada tres semanas.

A partir del cuarto ciclo mostró aumento del diámetro longitudinal y elevación de las lesiones en región inguinales de consistencia dura, no pétrea, no dolorosa, sin prurito, sin deterioro de la clase funcional. Debido al adecuado estado funcional y buena tolerancia se decide continuar con el tratamiento. 

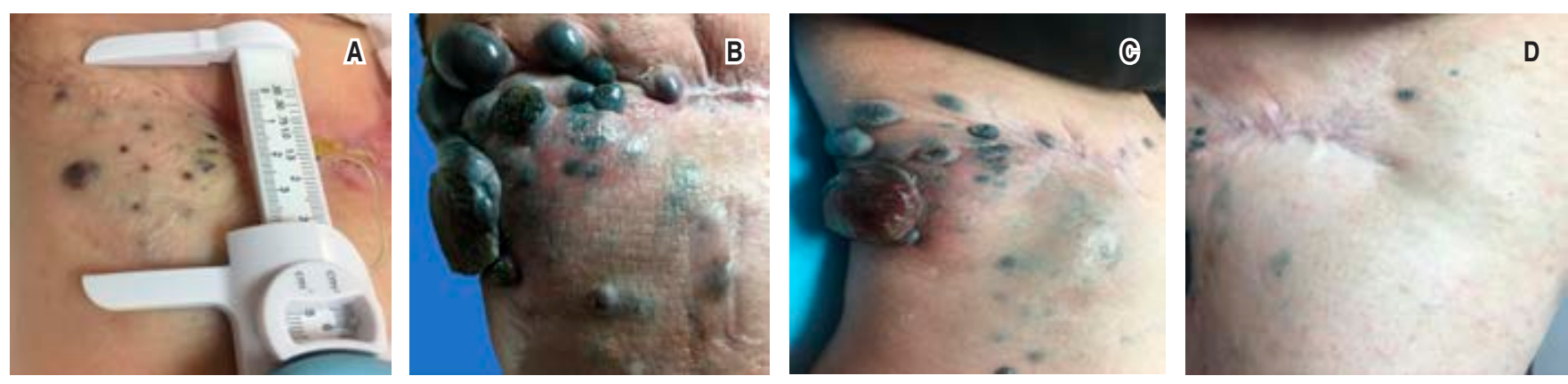

Figura 1: Pseudoprogresión cutánea. A) Lesión al diagnóstico. B) Pseudoprogresión máxima al tercer mes del tratamiento. C) Disminución de la lesión. D) 18 meses de tratamiento.

Cuatro aplicaciones después, presentó disminución de los diámetros de las lesiones, consistencia ahulada y finalmente blanda, por lo que se consideró pseudoprogresión del melanoma (Figura 1).

Durante la evaluación de la respuesta por imagen al año sólo con persistencia de la lesión pulmonar y actividad metabólica (PET CT), fue tratada con radiocirugía en el nódulo pulmonar. A la fecha, la paciente ha recibido 22 ciclos con respuesta parcial, tolerando adecuadamente el tratamiento y no ha presentado otras toxicidades.

\section{DISCUSIÓN}

El melanoma avanzado es la neoplasia de la piel más agresiva, con un mal pronóstico y clásicamente una respuesta insuficiente a la quimioterapia. Con el inicio del uso terapéutico de los anticuerpos monoclonales con mecanismo de acción sobre los ICIs y el bloqueo de las alteraciones de BRAF, el panorama para esta neoplasia cambió de manera sustancial. ${ }^{1}$

Con el uso cada vez más generalizado de estos biológicos, debemos reconocer que los patrones de respuesta que se establecieron en la época de los ensayos clínicos con quimioterapia no son suficientes para evaluar la respuesta a la inmunoterapia con los ICIs. ${ }^{9}$ En este caso, se describe una respuesta conocida como "pseudoprogresión", donde en un inicio hay incremento del diámetro de las lesiones para después presentar una reducción significativa y clínicamente relevante del volumen tumoral total. ${ }^{10}$

Este nuevo tipo de respuesta se ha observado en los ensayos clínicos de diferentes tumores sólidos, y en melanoma, en pacientes con inhibidores del eje PD1/PD-L1 se ha reportado una frecuencia de este fenómeno de entre 3 y $9 \% .{ }^{11}$ En un análisis conjunto de la FDA después de revisar más de 2,500 pacientes tratados con inmunoterapia se reportó $4 \%$ de pseu- doprogresión con una supervivencia global significativamente mayor contra los que no continuaron tratamiento más allá de la progresión de 24.4 versus 11.2 meses ${ }^{12}$ logrando correlacionar este evento de pseudoprogresión con un desenlace clínico diferente de los que claramente progresaron y también diferente de quienes tuvieron una respuesta objetiva inicial como primera respuesta. Se hizo evidente que los criterios de RECIST v1.1 no fueron capaces de evaluar estás nuevas formas de respuesta, lo que motivó la descripción de nuevas y numerosas formas de evaluar la respuesta ante la inmunoterapia. ${ }^{13-15}$

Los métodos actuales utilizados son: tomar una biopsia, considerada el estándar de oro, donde se identifica mayor infiltración del tejido por CD8 +, TIA1 + y granzima B +, en lugar de células tumorales viables, con el inconveniente de que es un método invasivo, por lo que se han buscado métodos no invasivos como los estudios radiológicos, los cuales identifican un aumento en el tamaño o número de lesiones con subsecuente disminución en el seguimiento.

Han surgido amplios debates sobre la fisiopatología de esta respuesta, pero no se ha alcanzado su comprensión total. ${ }^{16,17}$ Los estudios de patología de estos tumores en crecimiento mostraron un extenso infiltrado de células inmunes, necrosis y no células tumorales viables. ${ }^{18,19}$ Otros casos han reportado hemorragia, edema e infiltrados de células inmunes. ${ }^{20}$ Esto pone en claro que el tratamiento con los ICIs reactiva el reconocimiento que se da entre las células inmunes y las células tumorales, produciendo una respuesta citotóxica y reclutamiento de más células inmunes. Actualmente se busca identificar factores predictivos de sensibilidad o resistencia a la inmunoterapia para optimizar la selección de pacientes sin tener opciones reales en la clínica. El estándar para la identificación de una pseudoprogresión sería la biopsia de nuevas lesiones o las que están incremen- 
tando de tamaño, pero no siempre es factible, seguro o práctico. Se han explorado nuevos métodos de imagen como la resonancia magnética con el uso de un contraste llamado nanopartículas supermagnéticas de óxido de hierro, que en la secuencia T2, identifica inflamación, tejido necrótico y edema; asimismo se ha buscado correlacionar la captación por PET-TC con 18FDG, donde se evalúa un menor metabolismo general de las lesiones. También la disminución del ADN circulante tumoral por medio de biopsia líquida parece correlacionar con una pseudoprogresión en el contexto de lesiones incrementando de tamaño; los niveles séricos de IL-8 o el estado funcional del paciente también se han propuesto como indicadores de una progresión real o no; sin embargo, no existe información validada prospectiva que se pueda llevar a la práctica habitual. ${ }^{11}$

\section{CONCLUSIÓN}

La pseudoprogresión es un tipo de respuesta poco frecuente en pacientes con melanoma en tratamiento con inhibidores de puntos de control inmunológicos. Representa un desafío para el médico oncólogo y debe de ser evaluada antes de considerar un cambio de tratamiento.

Este caso ejemplifica una pseudoprogresión por su expresión clínica dermatológica que facilitó su seguimiento de manera estrecha sin necesidad de realizar estudios invasivos, y pone de manifiesto que es esencial identificar tempranamente este fenómeno en nuestros pacientes para no suspender el tratamiento de manera innecesaria o temprana.

\section{REFERENCIAS}

1. Schadendorf D, van Akkooi ACJ, Berking C, Griewank KG, Gutzmer R, Hauschild A et al. Melanoma. Lancet. 2018; 392 (10151): 971-984. doi: 10.1016/S0140-6736(18)31559-9.

2. Hartman RI, Lin JY. Cutaneous melanoma-a review in detection, staging, and management. Hematol Oncol Clin North Am. 2019; 33 (1): 25-38. doi: 10.1016/j.hoc.2018.09.005.

3. Hernández D, Aldaco F, Cervantes G, Erazo-Valle-Solís AA, Pérez-Pérez P, Torrecillas-Torres L et al. La mortalidad por melanoma en México aumenta. 2019; 23: 123-129.

4. Gallegos Hernández JF, Nieweg OE. Cutaneous melanoma (CM): current diagnosis and treatment. Gac Med Mex. 2014; 150 (Suppl 2): 175-182.

5. Camacho C, Gerson R, Góngora M, Villalobos A, Blanco YC, López O. Actualidades para el tratamiento del melanoma metastásico, estado del arte. An Med Asoc Med Hosp ABC. 2017; 62 (3): 196-207.

6. Robert C, Thomas L, Bondarenko I, O’Day S, Weber J, Garbe $\mathrm{C}$ et al. Ipilimumab plus dacarbazine for previously untreated metastatic melanoma. N Engl J Med. 2011; 364 (26): 25172526. doi: 10.1056/NEJMoa1104621.
7. Robert C, Ribas A, Schachter J, Arance A, Grob JJ, Mortier $\mathrm{L}$ et al. Pembrolizumab versus ipilimumab in advanced melanoma (KEYNOTE-006): post-hoc 5-year results from an open-label, multicentre, randomised, controlled, phase 3 study. Lancet Oncol. 2019; 20 (9): 1239-1251. doi: 10.1016/ S1470-2045(19)30388-2.

8. Robert C, Long GV, Brady B, Dutriaux C, Maio M, Mortier L et al. Nivolumab in previously untreated melanoma without BRAF mutation. N Engl J Med. 2015; 372 (4): 320-330. doi: 10.1056/NEJMoa1412082.

9. Daud AI. Revisiting RECIST: the case of treatment beyond progression. Lancet Oncol. 2018; 19 (2): 157-159. doi: 10.1016/ S1470-2045(18)30007-X.

10. Chiou VL, Burotto M. Pseudoprogression and immune-related response in solid tumors. J Clin Oncol. 2015; 33 (31): 35413543. doi: 10.1200/JCO.2015.61.6870.

11. Jia W, Gao Q, Han A, Zhu H, Yu J. The potential mechanism, recognition and clinical significance of tumor pseudoprogression after immunotherapy. Cancer Biol Med. 2019; 16 (4): 655-670. doi: 10.20892/j.issn.20953941.2019.0144.

12. Beaver JA, Hazarika M, Mulkey F, Mushti S, Chen H, He $\mathrm{K}$ et al. Patients with melanoma treated with an anti-PD-1 antibody beyond RECIST progression: a US Food and Drug Administration pooled analysis. Lancet Oncol. 2018; 19 (2): 229-239. doi: 10.1016/S1470-2045(17)30846-X.

13. Hodi FS, Hwu WJ, Kefford R, Weber JS, Daud A, Hamid O et al. Evaluation of immune-related response criteria and RECIST v1.1 in patients with advanced melanoma treated with pembrolizumab. J Clin Oncol. 2016; 34 (13): 1510-1517. doi: 10.1200/JCO.2015.64.0391.

14. Seymour L, Bogaerts J, Perrone A, Ford R, Schwartz LH, Mandrekar S et al. RECIST working group. iRECIST: guidelines for response criteria for use in trials testing immunotherapeutics. Lancet Oncol. 2017; 18 (3): e143-e152. doi: 10.1016/S1470-2045(17)30074-8.

15. Le Lay J, Jarraya H, Lebellec L, Penel N. irRECIST and iRECIST: the devil is in the details. Ann Oncol. 2017; 28 (7): 1676-1678. doi: 10.1093/annonc/mdx168. PMID: 28383646.

16. Nishino M, Giobbie-Hurder A, Manos MP, Bailey N, Buchbinder EI, Ott PA et al Immune-related tumor response dynamics in melanoma patients treated with pembrolizumab: identifying markers for clinical outcome and treatment decisions. Clin Cancer Res. 2017; 23: 4671-4679. doi: 10.1158/1078-0432.CCR-17-0114.

17. Katz SI, Hammer M, Bagley SJ, Aggarwal C, Bauml JM, Thompson JC et al Radiologic pseudoprogression during anti-PD-1 therapy for advanced non-small cell lung cancer. J Thorac Oncol. 2018; 13 (7): 978-986. doi: 10.1016/j. jtho.2018.04.010.

18. Cohen JV, Alomari AK, Vortmeyer AO, Jilaveanu LB, Goldberg SB, Mahajan A et al Melanoma brain metastasis pseudoprogression after pembrolizumab treatment. Cancer Immunol Res. 2016; 4: 179-182. doi: 10.1158/2326-6066.CIR15-0160.

19. Rocha P, Hardy-Werbin M, Naranjo D, Taus Á, Rodrigo M, Zuccarino F et al CD103+CD8+ lymphocytes characterize the immune infiltration in a case with pseudoprogression in squamous NSCLC. J Thorac Oncol. 2018; 13: e193-196. doi: 10.1016/j.jtho.2018.05.008.

20. Hochmair MJ, Schwab S, Burghuber OC, Krenbek D, Prosch $\mathrm{H}$ Symptomatic pseudo-progression followed by significant treatment response in two lung cancer patients treated with immunotherapy. Lung Cancer. 2017; 113: 4-6. doi: 10.1016/j. lungcan.2017.08.020. 